\section{Tumor Vascular Disrupting Agents}

\section{Neuartiges Wirkprinzip}

Die Substanz ASA404 hat in klinischen Studien der Phase II bei Patienten mit nichtkleinzelligem Lungenkarzinom in Kombination mit Paclitaxel und Carboplatin bereits erste Wirksamkeit bei guter Verträglichkeit gezeigt. Sollten sich diese Ergebnisse in der soeben begonnenen Studie ATTRACT-1 bestätigen, würde das den Weg für die Einfuihrung einer Substanz mit völlig neuartigem Wirkmechanismus ebnen.

Als Tumor Vascular Disrupting Agents (TVDAs, Tumorgefäß zerstörende Substanzen) bezeichnet man eine neue Klasse potenzieller Tumortherapeutika, deren spezifische AntiTumor-Aktivität auf morphologischen und molekularen Unterschieden zwischen etablierten tumorversorgenden Blutgefäßen und Gefäßen gesunder Organe beruht. Der Wirkmechanismus der T-VDAs unterscheidet sich damit grundlegend von etablierten antiangiogenen Substanzen wie Bevacizumab, Sunitinib und Sorafenib. Während diese antiangiogenen Substanzen über eine Hemmung der endothelialen Proliferation und Zellmigration und damit vor allem auf die peripheren Anteile des Tumors wirken, greifen T-VDAs selektiv an Endothelzellen von Tumorversorgenden Blutgefäßen an, unterbrechen den Blutfluss zum Tumorzentrum und induzieren dadurch Hypoxie sowie ausgedehnte hämorrhagische Nekrosen.

\section{Synergie durch Kombination mit Chemotherapie?}

Für die Therapie stark vaskularisierter, ausgedehnter solider Tumoren sind die T-VDAs möglicherweise besser geeignet als Angiogenesehemmer, da diese Tumoren bereits über ein etabliertes Gefäßsystem verfügen und das Überleben des Tumors weniger stark von der Gefäßneubildung abhängt als bei kleineren Tumoren bzw. in einem früheren Stadium des Tumorwachstums.

Die Wirkung der T-VDAs auf das Tumorzentrum wird als günstige Voraussetzung für Synergien mit chemotherapeutischen Ansätzen angesehen, da diese primär auf die Tumorperipherie wirken. Auch die jeweils eigenständigen Wirkmechanismen von T-VDAs und Chemotherapie können sich in ihrer Antitumorwirkung gut ergänzen und zur Verhinderung überlappender Toxizitäten beitragen.

\section{Niedrigmolekularer Wirkstoff mit einmaligem Wirkmechanismus}

Bei den derzeit untersuchten T-VDAs unterscheidet man zwischen Tubulinantagonisten wie AVE8062A, Combretastatin-A4-Phosphat (CA4P) oder ZD6126 auf der einen und dem Flavonoid ASA404 auf der anderen Seite. Bei ASA404 handelt es sich um den niedrigmolekularen Wirkstoff 5,6-Dimethyl-Xanthenone4-Acetic Acid (DMXAA) mit einem bisher einmaligen, tubulin-unabhängigen, dualen Wirkmechanismus [1]. Dieser umfasst einen direkten Apoptose-induzierenden Effekt auf Endothelzellen mit konsekutiver Thrombozytenaktivierung und Serotonin (5-HT)-Freisetzung sowie einen indirekten Effekt über die Ausschüttung weiterer vasoaktiver Substanzen wie Tumor Nekrose Faktor (TNF) und Stickstoffmonoxid (NO) (Abb. 1). Die beschriebenen Mechanismen bewirken im Tumorzentrum eine hämorrhagische Nekrose und nachfolgend eine Hypoxie des Tumorgewebes.
$1200 \mathrm{mg} / \mathrm{m}^{2}$ als optimale biologische Dosis

Im Rahmen des klinischen Entwicklungsprogramms zu ASA404 wurden zunächst 2 Dosiseskalationsstudien an insgesamt 108 Patienten durchgeführt. Die dabei ermittelte, maximal tolerierte, 3-wöchentliche i.v.-Dosis liegt bei $3700 \mathrm{mg} / \mathrm{m}^{2}$ Körperoberfläche. Nebenwirkungen, die in dieser Dosierung auftraten, waren unter anderem Sehstörungen und QTc-Verlängerung. Dosislimitierende Nebenwirkungen, die zusätzlich bei der maximal eingesetzten Dosierung von $4900 \mathrm{mg} / \mathrm{m}^{2}$ auftraten, waren unter anderem Linksherzversagen. Hämatologische Toxizitäten wurden auch bei maximaler Dosierung nicht beobachtet [2].

In der darauf folgenden Phase-I-Studie zur Sicherheit von ASA404 wurden 6 Dosierungen zwischen wöchentlich $300 \mathrm{mg} / \mathrm{m}^{2}$ und $3000 \mathrm{mg} /$ $\mathrm{m}^{2}$ an 15 Patienten erprobt. Kardiale Symptome wie unter anderem Verlängerungen der QTcZeit traten nur bei Dosierungen ab $2400 \mathrm{mg} / \mathrm{m}^{2}$ auf. Vorübergehende Blutdrucksteigerungen erwiesen sich als dosisabhängig. Visusbeeinträchtigungen wurden unter keiner der geprüften Dosierungen beobachtet. Basierend auf den PhaseI-Daten wurde die Dosierung von $1200 \mathrm{mg} / \mathrm{m}^{2}$ als optimale biologische Dosis für die folgenden Phase-II-Studien empfohlen [3].

\section{Überlebensvorteil durch ASA404?}

Das fortgeschrittene NSCLC wird die erste Indikation bei einem zukünftigen Einsatz von ASA404 sein. Im Rahmen einer offenen, randomisierten, multizentrischen Studie wurden 73 Patienten mit Chemotherapie-naivem NSCLC im Stadium IIIlb oder IV behandelt [4]. Die Patienten erhielten eine Carboplatin/PaclitaxelStandardtherapie in 3-wöchentlichen Zyklen entweder alleine oder in Kombination mit ASA404 1200 mg/m². Nach RECIST-Kriterien (Response Evaluation Criteria in Solid Tumors) erreichten $22 \%$ der alleine mit der Stan-

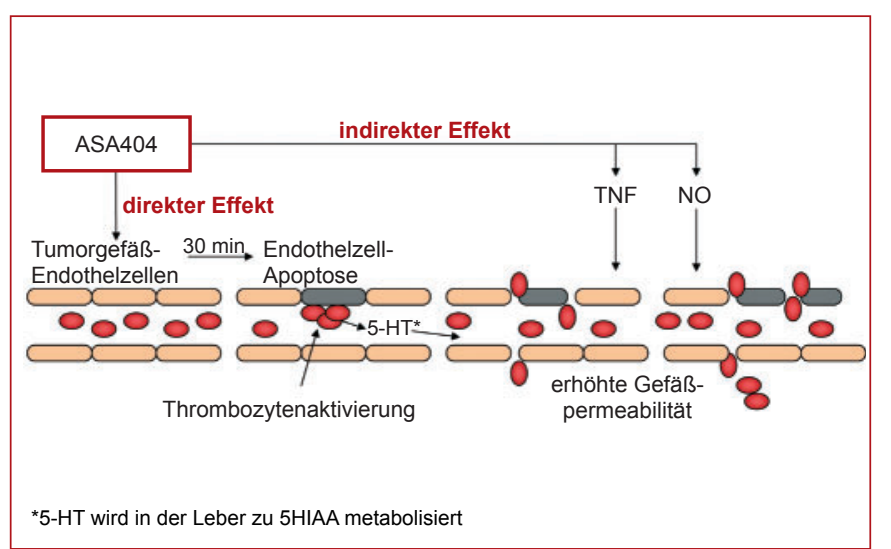

Abb. 1. Dualer Wirkmechanismus von ASA404 [1].

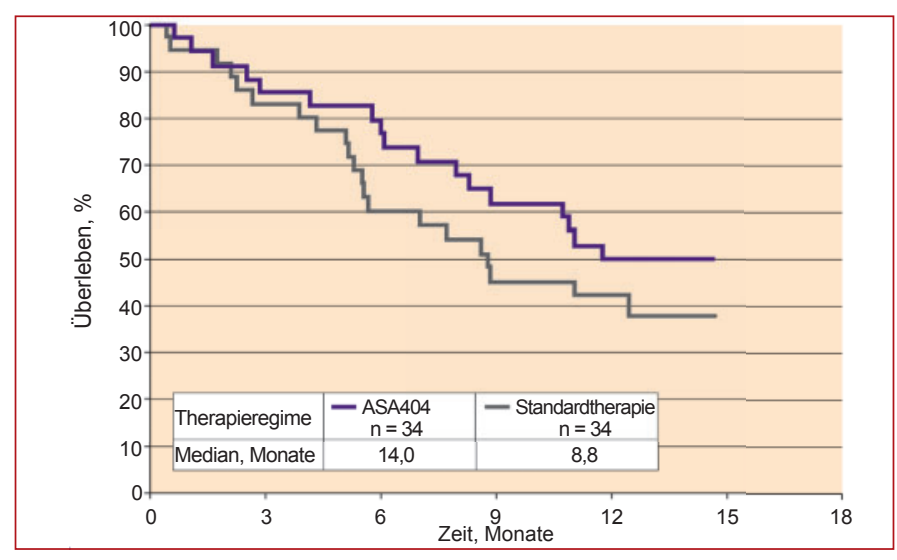

Abb. 2. Gesamtüberleben unter Chemotherapie mit und ohne ASA404 (DMXAA) $1200 \mathrm{mg} / \mathrm{m}^{2}$ [5]. 


\begin{tabular}{|c|c|c|c|c|c|c|}
\hline \multirow[b]{2}{*}{ Therapieregime } & \multicolumn{2}{|c|}{ Plattenepithel-NSCLC } & \multicolumn{2}{|c|}{ Nicht-Plattenepithel-NSCLC } & \multicolumn{2}{|l|}{ Alle } \\
\hline & $\mathrm{CP}$ & $\mathrm{CP}+\mathrm{ASA} 404$ & $\mathrm{CP}$ & $\mathrm{CP}+\mathrm{ASA} 404$ & $\mathrm{CP}$ & $\mathrm{CP}+\mathrm{ASA} 404$ \\
\hline $\begin{array}{l}\text { Anteil der Patienten mit Nebenwirkungen Grad } 3 \\
\text { oder höher (NCI-CTC*) }(\%)\end{array}$ & $\begin{array}{l}72,7 \\
(\mathrm{n}=11)\end{array}$ & $\begin{array}{l}68,2 \\
(\mathrm{n}=22)\end{array}$ & $\begin{array}{l}64,0 \\
(\mathrm{n}=25)\end{array}$ & $\begin{array}{l}60,9 \\
(n=46)\end{array}$ & $\begin{array}{l}66,7 \\
(\mathrm{n}=36)\end{array}$ & $\begin{array}{l}63,2 \\
(\mathrm{n}=68)\end{array}$ \\
\hline Ansprechrate $(\%)^{* *}$ & 14,3 & 40,0 & 25,0 & 31,7 & 22,2 & 34,4 \\
\hline $\begin{array}{l}\text { Mediane Zeit bis zur Tumorprogression } \\
\text { (Monate) }\end{array}$ & $\begin{array}{l}1,6 \\
(\mathrm{n}=11)\end{array}$ & $\begin{array}{l}5,6 \\
(n=21)\end{array}$ & $\begin{array}{l}4,8 \\
(n=25)\end{array}$ & $\begin{array}{l}5,5 \\
(n=43)\end{array}$ & $\begin{array}{l}4,4 \\
(n=36)\end{array}$ & $\begin{array}{l}5,5 \\
(n=64)\end{array}$ \\
\hline $\begin{array}{l}\text { Mediane Überlebenszeit } \\
\text { (Monate) }\end{array}$ & $\begin{array}{l}5,5 \\
(\mathrm{n}=11)\end{array}$ & $\begin{array}{l}10,2 \\
(\mathrm{n}=21)\end{array}$ & $\begin{array}{l}11,0 \\
(\mathrm{n}=25)\end{array}$ & $\begin{array}{l}14,9 \\
(\mathrm{n}=43)\end{array}$ & $\begin{array}{l}8,8 \\
(\mathrm{n}=36)\end{array}$ & $\begin{array}{l}14,5 \\
(\mathrm{n}=64)\end{array}$ \\
\hline
\end{tabular}

*National Cancer Institute Common Toxicity Criteria, Skala von 0-4

** Bei 12 Patienten war zum Parameter «Ansprechrate» keine Auswertung möglich

Tab. 1. Subgruppenanalyse der Phase-II-Studien mit ASA404: Plattenepithel- vs. Nicht-Plattenepithel-NSCLC [6].

dard-Chemotherapie und 31\% der zusätzlich mit ASA404 behandelten Patienten eine partielle Remission. Die mediane Zeit bis zur Tumorprogression betrug 5,4 Monate in der ASA404-Gruppe und 4,4 Monate in der Standardtherapie-Gruppe. Die mediane Gesamtüberlebenszeit der Patienten, die ASA404 erhalten hatten, lag bei 14 Monaten. Unter der Standardtherapie betrug sie 8,8 Monate (Abb. 2).

Eine einarmige Phase-II-Studie bestätigte die Wirksamkeit und Sicherheit der Kombination von Standardchemotherapie und ASA404 in einer Dosierung von $1800 \mathrm{mg} /$ $\mathrm{m}^{2}$ alle 3 Wochen. 11 von insgesamt $29 \mathrm{~Pa}-$ tienten $(38 \%)$ erreichten eine partielle $\mathrm{Re}$ mission. Die mediane Zeit bis zur Tumorprogression lag bei 5,5 Monaten, die mediane Überlebenszeit bei 14,9 Monaten [5]. In einer gepoolten Analyse der beiden Studien verglichen McKeage und Jameson [6] Wirksamkeit und Verträglichkeit von ASA404 bei Patienten mit Plattenepithel-NSCLC gegenüber Nicht-Plattenepithel-NSCLC. In beiden Subgruppen erwies sich die Kombination von ASA404 und Chemotherapie als der alleinigen Chemotherapie überlegen. Insgesamt konnte bei der gesamten Patientengruppe die mediane Überlebenszeit von 8,8 Monate (CP) auf 14,4 Monate (CP+ASA404) verbessert werden. Die Verträglichkeit der beiden Therapieregimes war in den beiden histologischen Subgruppen vergleichbar (Tab. 1) [4].

\section{Verträglich auch in Kombination mit Chemotherapie}

Pharmakologische Interaktionen zwischen ASA404 und Chemotherapie wurden bisher nicht beobachtet. Die Rate schwerwiegender Nebenwirkungen betrug unter der Standardchemotherapie $47 \%$ und bei den Patienten, die zusätzlich ASA404 erhalten hatten, 43\%. Infektionen, kardiale Symptome und Allgemeinsymptome wie Irritationen an der Injektionsstelle traten bei mehr als 10\% der Patienten auf, die ASA404 erhalten hatten. ASA404 zeigte keinen Einfluss auf das QTc-Intervall. Kardiale Beschwerden erwiesen sich in allen
Fällen als vorübergehend, reversibel und standen nach Einschätzung der behandelnden Ärzte nicht mit der Studienmedikation in Verbindung. Die Ergebnisse zur Sicherheit von ASA404 wurden in der Studie von McKeage et al. [5] für die dreiwöchentliche Gabe von $1800 \mathrm{mg} / \mathrm{m}^{2}$ bestätigt. Schwerwiegende $\mathrm{Ne}$ benwirkungen traten bei $48 \%$ der Patienten auf, darunter keine kardialen Effekte. Unter den schwerwiegenden Nebenwirkungen waren Neutropenie und periphere Neuropathien mit einer Inzidenz $\geq 10 \%$ am häufigsten. Die ophthalmologische Untersuchung zeigte in beiden Studien keine bedeutsamen therapiebedingten Auffälligkeiten. Nebenwirkungen, die für antiangiogene Substanzen typisch sind, wie arterielle Thrombosen, Hypertonie, Proteinurie, Wundheilungs- oder Blutgerinnungsstörungen, wurden in diesen beiden Studien unter ASA404 nicht beobachtet.

\section{ATTRACT-1-Rekrutierung begonnen}

Diese vielversprechenden Phase-II-Daten beim NSCLC bilden die Grundlage für das gerade gestartete Studienprogramm ATTRACT (Antivascular Targeted Therapy: Researching ASA404 in Cancer Treatment). Zunächst sollen für die internationale, randomisierte und doppelblinde PhaseIII-Studie ATTRACT-1 1200 Patienten mit Chemotherapie-naivem NSCLC Stadium IIIb oder IV rekrutiert werden. Alle Patienten erhalten in 3-wöchigen Zyklen eine Standardchemotherapie mit Paclitaxel und Carboplatin sowie zusätzlich entweder ASA404 1800 mg/ $\mathrm{m}^{2}$ oder Plazebo. Mit der internationalen Rekrutierung wurde jetzt in Neuseeland erfolgreich begonnen. Der Rekrutierungsstart in Deutschland ist für Sommer 2008 geplant. Insgesamt sollen Patienten an 18 deutschen Zentren betreut werden. Studienleiter für Deutschland ist Dr. Ulrich Gatzemeier vom onkologischen Schwerpunkt des Krankenhauses Großhansdorf.

Bestätigen sich die vielversprechenden Ergebnisse der Phase-I- und -II-Studien in ATTRACT-1, könnte mit ASA404 bald ein völlig neues Wirkprinzip in die Onkologie eingeführt werden.

\section{Dr. Thomas Bißwanger-Heim, Freiburg}

Weitere Informationen zur Phase-III-Studie ATTRACT-1 erhalten Sie über:

Dr. med. Ulrich Gatzemeier

Krankenhaus Großhansdorf GmbH

Zentrum für Pneumologie und Thoraxchirurgie

Onkologischer Schwerpunkt

Wöhrendamm 80

22927 Großhansdorf

Tel.: +49 $4102601-260$

E-mail:pneumo.onko@t-online.de

\section{Referenzen}

1 Baguley BC: Antivascular therapy of cancer: DMXAA Lancet Oncol 2003;4:141-8.

2 McKeage MJ, Kelland LR: 5,6-Dimethylxanthenone-4 Acetic Acid (DMXAA): Clinical Potential in Combination with Taxane-Based Chemotherapy. Am J Cancer 2006;5:155-62. 3 McKeage MJ, Fong P, Jeffery M, Ravic M, Jameson MB DART - a phase I safety and dose-finding study of the vascular targeting agent 5,6-dimethylxanthenone-4-acetic acid (DMXAA) in the treatment of refractory tumors. J Clin Oncol 2005;23(16, suppl):212s. Abstract 3081.

4 Pawel J, Reck M, McKeage M, AS1404-201 Study Group Investigators: Update on survival in a phase $\mathrm{Ib} / \mathrm{II}$ study of DMXAA (AS1404) combined with carboplatin and paclitaxel in non-small cell lung cancer (NSCLC). J Clin Oncol 2007;25: S18-S5115.

5 McKeage MJ, von Pawel J: Phase II study of DMXAA (ASA404) $1800 \mathrm{mg} / \mathrm{m}^{2}$ combined with carboplatin and paclitaxel in non-small cell lung cancer. J Thorac Oncol 2007;2(8, Suppl. 4): Abst P3-116.

6 McKeage MJ, Jameson MB, AS1404-201 Study Group Investigators: Comparison of safety and efficacy between squamous and non-squamous non-small cell lung cancer (NSCLC) patients in phase II studies of DMXAA (ASA404). J Clin Oncol 2008;26:(May 20 suppl; abstr 8072).

\section{Impressum}

Tumor Vascular Disrupting Agents Neuartiges Wirkprinzip

PharmaForum in ONKOLOGIE 31 | 7 | 08

C 2008 by S. Karger Verlag für Medizin und Naturwissenschaften $\mathrm{GmbH}$

Lörracher Straße 16a

79115 Freiburg, Deutschland

Mit freundlicher Unterstützung durch Novartis Pharma GmbH.

Der Verlag und die Herausgeber der Zeitschrift übernehmen keine Verantwortung für diese Rubrik.

\section{KARGER (을 2008 S. Karger GmbH, Freiburg}

